\title{
Review
}

\section{Medical Mycology Seen Through a One Health Approach}

\author{
Hiroshi Kakeya ${ }^{1,2}$ \\ ${ }^{1}$ Department of Infection Control Science, Osaka City University Graduate School of Medicine \\ ${ }^{2}$ Research Center for Infectious Disease Sciences, Osaka City University, Graduate School of Medicine
}

\begin{abstract}
In clinical settings, the number of immune compromised patients have increased as a result of developments in medical technology (e.g., organ transplantation, anticancer drugs, steroids, TNF inhibitors, etc.). However, patients with fungal diseases are also increasing globally. In recent years, the distribution and pathogenicity of fungi worldwide have been changing, with reports that new fungi are emerging, and antifungal-resistant fungi are spreading globally. Global warming, globalization, human activities, and other factors have been suggested as contributing to the emergence of new fungi. Some of the antifungals against which resistant fungi have emerged are commonly used not only for human but also for animal health care and crop protection. Consequently, the occurrence of antifungal-resistant fungi has become a clinical issue. Solving these problems entails continuing the "One Health" approach, which in turn requires updating medical mycology information with regard to the emerging pathogenic fungi. In particular, this paper reviews the recent information on Cryptococcus gattii, Candia auris, and azoleresistant Aspergillus fumigatus.
\end{abstract}

Key words : azole-resistant Aspergillus fumigatus, Candida auris, Cryptococcus gattii

\section{Introduction}

Currently, more than 600 fungal species are known to cause infections in humans. In recent years, the distribution and pathogenicity of fungi worldwide have been changing, and the frequency of fungal infections in animals and plants has been increasing. In particular, plant diseases caused by fungi are threatening to cause species extinction, leading to food shortages. Global warming, globalization, and other factors are contributing to these problems ${ }^{1}$.

Among the emerging fungal diseases, those caused by Cryptococcus gattii, a new species that appeared on Vancouver Island, British Columbia, Canada in 1999, and Candida auris, which was discovered in Japan in 2009 and has since become a clinical concern worldwide, are noteworthy. Antimicrobial resistance has also become a worldwide problem, leading to the promotion of antimicrobial stewardship programs. Trends in antifungal drug resistance have also become of concern. The U.S. Centers for Disease Control and Prevention (CDC) 2019 Antibiotic Resistance Threats Report ${ }^{2)}$ lists drug-resistant $\mathrm{Ca}$. auris among the urgent threats and drug-resistant Candida spp. among the serious threats; while azole-resistant Aspergillus fumigatus is on its watch list.

This article addresses $C r$. gattii, $C a$. auris, and azoleresistant A. fumigatus as fungi of interest in the last two decades and reviews the causes of their emergence and spread from a "One Health" perspective.

\section{Cryptococcus gattii}

Cr. gattii is found in eucalyptus trees in tropical and subtropical regions such as Papua New Guinea and Australia and is mainly known to be a pathogen of koalas, and human infections are rare. However, starting with an outbreak on Vancouver Island, British Columbia, Canada in 1999, 218 cases of human infections were reported over an eight-year period through 2007, with a high average incidence of 27.9 cases per 1 million inhabitants on Vancouver Island between 2002 and $2006^{3)}$. It then spread to the adjacent U.S. Pacific coast (Washington, Oregon, and California), where 96 cases were reported between 2004 and 2011 $1^{4)}$.

Cr. gattii is further subdivided into four genotypes; namely, VGI to VGIV (VGII is further subdivided into VGIIa to

Address for correspondence: Hiroshi Kakeya, MD, PhD

Department of Infection Control Science, Osaka City University Graduate School of Medicine, 1-4-3 Asahi-machi, Abeno-ku, Osaka 545-8585, Japan Received: 13 September 2021, Accepted: 24 September 2021

E-mail: kakeya@med.osaka-cu.ac.jp 
VGIIc), with VGI being widely distributed in Australia, Papua New Guinea, Asia, and Europe. VGII is prevalent in North and South America, particularly in British Columbia, where the outbreak of VGIIa occurred, and VGIIa, VGIIb, and VGIIc are found on the Pacific coast of the U.S., especially VGIIc, which is a new genotype that has not been reported outside the U.S. ${ }^{5,6}$. It has been determined that VGIIa and VGIIc are highly pathogenic ${ }^{7}$.

\section{Human-mediated $\boldsymbol{C r}$. gattii dispersal}

In British Columbia, where $C r$. gattii is not prevalent, it has been detected in the tires of $35 \%$ (22/63) of cars. A total of 8 million cars travel between Vancouver Island and the BC Mainland every year, suggesting that the fungus was carried by car tires. Cr. gattii was also detected in 54\% (43/80) of footwear (confirmed to enable survival up to 333 days), suggesting that it may have spread due to human activity ${ }^{8)}$.

It has been contended that the spores of $\mathrm{Cr}$. gattii detected on Vancouver Island are smaller than those of plant origin, which may have facilitated the spread of the infection, that same-sex mating of Cryptococcus may have occurred and altered its characteristics, and that habitat expansion may be associated with global environmental changes such as global warming ${ }^{9)}$.

Cr. gattii (VGII) has also been detected in cats with cutaneous cryptococcosis in an urban area in Brazil, suggesting that the spread of $\mathrm{Cr}$. gattii can occur through animals ${ }^{10)}$. These reports suggest the spread of new Cryptococcus genotypes. Cr. gattii infections involve human and animal activities; thus, emerging fungal diseases should be considered from the perspective of a One Health approach in the future. Cr. gattii infections in Japan

Cases in Japan are rare. A male patient in Tokyo with underlying diabetes mellitus presented with headache and right homonymous hemianopia and underwent craniotomy due to a mass of approximately $4 \mathrm{~cm}$ in size that was recognized in the left frontal lobe on a head computed tomography (CT) scan. Brain lesions caused by $C r$. gattii were diagnosed by histopathological examination and genetic testing of the strain obtained through culture ${ }^{11}$.

In another case, a man in Aichi Prefecture with no underlying disease presented with headache and low-grade fever, and bronchoscopy and cerebrospinal fluid examination were performed as the chest CT scan revealed a mass shadow of approximately $5 \mathrm{~cm}$ in size in the right lower lobe, and head MRI revealed multiple brain lesions. Histopathology of the transbronchial lung biopsy and the cerebrospinal fluid by India ink revealed yeast-like fungi suggestive of Cryptococcus, and heterogeneity testing of the strain obtained in culture led to the diagnosis of disseminated cryptococcosis due to $\mathrm{Cr}$. gattii $^{12}$.

\section{Candida auris}

Ca. auris is a new species of the genus Candida described by Sato et al. in $2009^{13}$. Of the 1468 strains collected from all over Japan by the Japan Antifungal Surveillance Program (JASP), only one strain, which could not be identified, was determined to be a new species by molecular phylogenetic analysis. Since this strain was detected in the ear canal discharge of a patient, it was named Ca. auris, after the Latin word "auris", which means "ear".

Originally, Ca. auris was not noted clinically because of its low detection rate and preserved antifungal susceptibility; however, in 2009, it was reported in a Korean patient with otitis media $^{14)}$. In 2011, it was reported in three hospital-onset fungal infections ${ }^{15}$. These strains showed reduced susceptibility to fluconazole (FLCZ). Transmission and epidemics have been reported in India ${ }^{16)}$, South Africa ${ }^{17}$, the United States ${ }^{18,19)}$, and Europe ${ }^{20)}$. Moreover, recent reports have shown that some strains have poor susceptibility to antifungals such as amphotericin B (AMPH-B), voriconazole (VRCZ), and caspofungin, and some strains are multidrug-resistant. Thus, the existence of multidrug-resistant strains, the difficulty in identifying species by standard laboratory assays, and the need for identification by molecular biological techniques and mass spectrometry have been reported, in addition to three cases of local colonization and outbreaks in healthcare facilities. Therefore, Ca. auris infection is now a major public health issue.

In an epidemiological study of Ca. auris in Japan (2019) ${ }^{21)}$, only 3 of the 13 isolates, all of which were isolated from the periauricular region, were resistant to azoles (FLCZ and VRCZ), and all isolates remained susceptible to AMPH-B and echinocandins. Thus, multidrug-resistant $\mathrm{Ca}$. auris is currently not a clinical concern in Japan. However, because Ca. auris possesses a high biofilm-forming ability, has high environmental viability and high transmissibility, and multiple clades exist worldwide, there is a risk of colonization by multidrugresistant $\mathrm{Ca}$. auris in the future and the possibility that the East Asian-type of $\mathrm{Ca}$. auris may acquire secondary resistance in Japan. These trends should therefore be monitored.

$\mathrm{Ca}$. auris is broadly classified into East Asian (Japan/ Korea), South Asian (India/ Pakistan/ Malaysia), South African, and South American (Venezuela) types, with each clade having a different tendency toward antifungal resistance $^{22)}$. Ca. auris is currently spreading rapidly worldwide, and although it is likely that $\mathrm{Ca}$. auris has been established as infectious in Japan and Korea since around 2000 and elsewhere since the late 2000 s, it is likely that it emerged simultaneously in different regions rather than solely from East Asia, which is thought to have been the region of its first occurrence. 
The cause of the nearly simultaneous emergence of each genetically distinct clade on three continents is unknown; however, it has been suggested that azoles present in the environment may be involved in the acquisition of resistance and that the possible acquisition of temperature tolerance may be involved in the acquisition of human pathogenicity ${ }^{23)}$.

Regarding the origin of pathogenic $\mathrm{Ca}$. auris, Casadevall et al. cited factors such as global warming, multidrug resistance due to HSP90 overexpression, changes in pathogenicity, and temperature sensitivity as reasons why $\mathrm{Ca}$. auris, traditionally considered an environmental fungus, has acquired pathogenicity in humans ${ }^{24)}$.

\section{Azole-resistant Aspergillus fumigatus}

A. fumigatus, the causative fungus of aspergillosis, is originally susceptible to azoles, echinocandins, and AMPH-B; however, azole-resistant $A$. fumigatus has recently received increased attention ${ }^{25}$. There are two main strains of azoleresistant A. fumigatus: human-derived and environmentally derived strains. Human-derived strains are mainly detected in patients receiving long-term treatment with azoles. The longterm use of azoles induces genetic mutations in the target enzyme Cyp51A, resulting in resistance to the drug. Some strains are not cross-resistant to other azoles (e.g., resistant to itraconazole and susceptible to VRCZ) depending on the site of the mutation. In contrast, among environmentally derived strains, a particular strain is known to have a mutation in the Cyp51A gene (L98H) with a 34-nucleotide tandem repeat sequence (TR34) in the promoter region. Environmentally derived strains with this resistance mechanism are resistant to more than one azole-based drug (multi-azole-resistant). Environmentally derived, azole-resistant strains were first reported in the Netherlands in $2008^{26}$; however, several azole analogs have been used as pesticides in Europe since the 1990s and have been suggested to be associated with the emergence of multi-azole-resistant A. fumigatus $^{27,28)}$. Initially, multiazole-resistant $A$. fumigatus was detected mainly in Europe; however, in recent years, it has also been detected in many other countries ${ }^{29}$. One of the possible reasons for this worldwide spread is that azole-resistant A. fumigatus may have adhered to and spread by tulip bulbs exported from the Netherlands ${ }^{30}$.

The azole drug VRCZ is the first recommended treatment for aspergillosis, whereas echinocandins and liposomal amphotericin B (L-AMB), which have different mechanisms of action, are used for the treatment of azole-resistant $A$. fumigatus. Therefore, in areas where azole-resistant $A$. fumigatus is isolated in more than $10 \%$ of cases, experts suggest opting for a combination of VRCZ and echinocandins or L-AMB as the initial treatment and changing antifungal drugs after confirming antifungal susceptibility outcomes ${ }^{31}$.
Isolation status of Japanese Azole-resistant $A$. fumigatus

In Japan, multi-azole-resistant A. fumigatus was first reported in a patient's specimen in 2016. However, this patient had the infection in 2013, suggesting that multi-azoleresistant strains had existed in Japan for some time ${ }^{32)}$. In 2017, multi-azole-resistant $A$. fumigatus was detected in Hokkaido ${ }^{33)}$. In regard to local epidemiology, it has been reported that $12.7 \%$ of A. fumigatus strains are resistant to azoles in the Kyoto and Shiga regions, and some multi-azole-resistant strains with the TR34/L98H mutation have been detected among them ${ }^{34}$. However, no national surveillance for the drug susceptibility of Aspergillus spp. has been conducted to date. Antifungal susceptibility testing should be conducted in each region to clarify the actual situation in Japan.

A causal investigation by Nakano et al. of azole-resistant Aspergillus imported into Japan found that 7.8\% (13/167) of A. fumigatus isolates from tulip bulbs imported from the Netherlands (Gladiolus, Hippeastrum, Tulipa, etc.) were azole-resistant but only found azole resistance in 1.2\% (1/86) of isolates from bulbs in Japan (Ipheion, Narcissus, Tulipa, etc.) ${ }^{35)}$.

These results suggest that drug-resistant fungi are being introduced into Japan via agricultural products imported from abroad.

At present, azole-resistant Aspergillus strains are not considered prevalent in Japan; however, it is important to keep an eye on future trends. Especially, azole-resistant organisms have been isolated from patients with long-term azole use ${ }^{36)}$. A nationwide survey is therefore necessary.

In filamentous fungal infections, antifungal drugs should ideally be selected on the basis of accurate species identification and antifungal susceptibility testing. In Japan, only a limited number of facilities are able to perform genetic identification and antifungal susceptibility testing for filamentous fungi. In addition, antifungal susceptibility testing for filamentous fungi is not covered by the Japanese health insurance system (as of September 2021). Antifungal susceptibility testing of filamentous fungi is also considered more time-consuming and more difficult than testing of yeasts; this is believed to be the reason why the test is not widely used. In the future, it will be necessary for laboratories to acquire competencies in antifungal susceptibility testing and establish a system for consultation with specialized facilities in each region.

In conclusion, these findings suggest that the evolution of antifungal resistance is caused by an increase in the number of susceptible patients due to population growth, urbanization, and immunosuppressive and anticancer drug treatments. In addition, there is a limited selection of antifungal drugs, wherein prophylaxis and empiric treatment with the same antifungal drugs is leading to the development of resistant fungi. Likewise, the movement of people and products with 
the development of transportation networks is leading to the dispersal of resistant fungi on a global scale ${ }^{37)}$. There is a limit to what we can do to influence population growth, urbanization, and the prevention of the dispersal of resistant strains and new emerging pathogenic fungi. What we can do is to search for the causative fungus, which is the basis of infectious disease treatment, confirm its antifungal susceptibility, and select the appropriate antifungal drug according to the condition and pathology of the host. The current state of hospitals, however, indicates that they do not have an adequate testing system in place. Proactive introduction of state-of-theart technology is also necessary to promote antifungal stewardship.

\section{Acknowledgments}

We would like to thank Editage (www. editage.jp) for English language editing.

\section{Conflicts of interest}

None.

This article was presented at $64^{\text {th }}$ Annual Meeting of the Japanese Society for Medical Mycology, Tokyo, in 2020.

\section{References}

1) Fisher MC, Henk DA, Briggs CJ, Brownstein JB, Madoff LC, McCraw SL, Gurr SJ: Emerging fungal threats to animal, plant and ecosystem health. Nature 484: 186-194, 2012.

2) U.S. Department of Health and Human Services, Centers for Disease Control and Prevention (CDC): Antibiotic resistance threats in the United States, 2019. Atlanta, GA, 2019. https: //www.cdc.gov/drugresistance/pdf/threats-report/2019-ar-threatsreport-508.pdf

3) BC Center for Disease Control: 2011 annual summary of reportable diseases. Vancouver, BC, Canada, 2011.

4) Harris JR, Lockhart SR, Debess E, Marsden-Haug N, Goldoft M, Wohrle R, Lee S, Smelser C, Park B, Chiller T: Cryptococcus gattii in the United States: clinical aspects of infection with an emerging pathogen. Clin Infect Dis 53: 11881195, 2011.

5) Galanis E, MacDougall L, Kidd S, Morshed M: Epidemiology of Cryptococcus gattii, British Columbia, Canada, 1999-2007. Emerg Infect Dis 16: 251-257, 2010.

6) Byrnes EJ 3rd, Bildfell RJ, Frank SA, Mitchell TG, Marr KA, Heitman J: Molecular evidence that the range of the Vancouver Island outbreak of Cryptococcus gattii infection has expand into the Pacific Northwest in the United States. J Infect Dis 199: 1081-1086, 2009.

7) Byrnes EJ 3rd, Li W, Lewit Y, Ma H, Voelz K, Ren P, Carter DA, Chaturvedi V, Bildfell RJ, May RC, Heitman J: Emergence and pathogenicity of highly virulent Cryptococcus gattii genotypes in the northwest United States. PLoS Pathog 6: e1000850, 2010.

8) Kidd SE, Bach PJ, Hingston AO, Mak S, Chow Y, MacDougall L, Kronstad JW, Bartlett KH: Cryptococcus gattii dispersal mechanisms, British Columbia, Canada. Emerg Infect Dis 13: 51-57, 2007.

9) Fraser JA, Giles SS, Wenink EC, Geunes-Boyer SG, Wright JR, Diezmann S, Allen A, Stajich JE, Dietrich FS, Perfect JR, Heitman J: Same-sex mating and the origin of the Vancouver Island Cryptococcus gattii outbreak. Nature 437: 1360-1364, 2005.

10) Brito-Santos F, Reis RS, Coelho RA, Almeida-Paes R, Pereira SA, Trilles L, Mayer W, Wanke B, Lazéra MDS, Gremião IDF: Cryptococcosis due to Cryptococcus gattii VGII in southeast Brazil: The One Health approach revealing a possible role for domestic cats. Med Mycol Case Rep 24: 61-64, 2019.

11) Okamoto $K$, Hatakeyama $S$, Itoyama $S$, Nukui $Y$, Yoshino $Y$, Kitazawa T, Yotsuyanagi H, Ikeda R, Sugita T, Koike K: Cryptococcus gattii genotype VGIIa infection in man, Japan, 2007. Emerg Infect Dis 16: 1155-1157, 2010.

12) Nakao M, Muramatsu H, Takahashi $T$, Niwa $S$, Kagawa $Y$, Kurokawa R, Sone K, Uozumi Y, Ohkusu M, Kamei K, Koga $\mathrm{H}$ : Cryptococcus gattii genotype VGIIa infection in an immunocompetent Japanese patient: a case report and minireview. Intern Med 55: 3021-3024, 2016.

13) Satoh K, Makimura K, Hasumi Y, Nishiyama Y, Uchida K, Yamaguchi H: Candida auris sp. nov., a novel ascomycetous yeast isolated from the external ear canal of an inpatient in a Japanese hospital. Microbiol Immunol 53: 41-44, 2009.

14) Kim MN, Shin JH, Sung H, Lee K, Kim EC, Ryoo N, Lee JS, Jung SI, Park KH, Kee SJ, Kim SH, Shin MG, Suh SP, Ryang DW: Candida haemulonii and closely related species at 5 university hospitals in Korea: identification, antifungal susceptibility, and clinical features. Clin Infect Dis 48: e57-61, 2009.

15) Lee WG, Shin JH, Uh Y, Kang MG, Kim SH, Park KH, Jang HC: First three reported cases of nosocomial fungemia caused by Candida auris. J Clin Microbiol 49: 3139-3142, 2011.

16) Chowdhary A, Sharma C, Duggal S, Agarwal K, Prakash A, Singh PK, Jain S, Kathuria S, Randhawa HR, Hagen F, Meis JF: New clonal strain of Candida auris, Delhi, India. Emerg Infect Dis 19: 1670-1673, 2013.

17) Magobo RE, Corcoran C, Seetharam S, Govender NP: Candida auris-associated candidemia, South Africa. Emerg Infect Dis 20: 1250-1251, 2014.

18) Calvo B, Melo AS, Perozo-Mena A, Hernandez M, Francisco EC, Hagen F, Meis JF, Colombo AL: First report of Candida auris in America: clinical and microbiological aspects of 18 episodes of candidemia. J Infect 73: 369-374, 2016.

19) Vallabhaneni S, Kallen A, Tsay S, et al: Investigation of the first seven reported cases of Candida auris, a globally emerging invasive, multidrug-resistant fungus-United States, May 2013-August 2016. Am J Transplant 17: 296-299, 2017.

20) Eyre DW, Sheppard AE, Madder H, et al: A Candida auris outbreak and its control in an intensive care setting. N Engl J Med 379: 1322-1331, 2018.

21) Iguchi S, Itakura $Y$, Yoshida A, Kamada K, Mizushima R, Arai Y, Uzawa Y, Kikuchi K: Candida auris: a pathogen difficult to identify, treat, and eradicate and its characteristics in Japanese strains. J Infect Chemother 25: 743-749, 2019.

22) Kathuria S, Singh PK, Sharma C, Prakash A, Masih A, Kumar 
A, Meis JF, Chowdhary A: Multidrug-resistant Candida auris misidentified as Candida haemulonii: characterization by matrix-assisted laser desorption ionization-time of flight mass spectrometry and DNA sequencing and its antifungal susceptibility profile variability by Vitek 2, CLSI broth microdilution, and Etest method. J Clin Microbiol 53: 1823-1830, 2015.

23) Rhodes J, Fisher MC: Global epidemiology of emerging Candida auris. Curr Opin Microbiol 52: 84-89, 2019.

24) Casadevall A, Kontoyiannis D, Robert V: On the Emergence of Candida auris: climate change, azoles, swamps, and birds. mBio 10: e01397-19, 2019.

25) Howard SJ, Cerar D, Anderson MJ, Albarrag A, Fisher MC, Pasqualotto AC, Laverdiere M, Arendrup MC, Perlin DS, Denning DW: Frequency and evolution of azole resistance in Aspergillus fumigatus associated with treatment failure. Emerg Infect Dis 15: 1068-1076, 2009.

26) Snelders E, van der Lee HA, Kuijpers J, Rijs AJ, Varga J, Samson RA, Mellado E, Donders AR, Melchers WJ, Verweij PE: Emergence of azole resistance in Aspergillus fumigatus and spread of a single resistance mechanism. PLoS Med 5: e219, 2008.

27) Snelders E, Camps SM, Karawajczyk A, Schaftenaar G, Kema GH, van der Lee HA, Klaassen CH, Melchers WJ, Verweij PE: Triazole fungicides can induce cross-resistance to medical triazoles in Aspergillus fumigatus. PLoS One 7: e31801, 2012.

28) Verweij PE, Snelders E, Kema GHJ, Mellado E, Melchers WJG: Azole resistance in Aspergillus fumigatus: a side-effect of environmental fungicide use? Lancet Infect Dis 9: 789-795, 2009.

29) Resendiz Sharpe A, Lagrou K, Meis JF, Chowdhary A, Lockhart SR, Verweij PE: Triazole resistance surveillance in Aspergillus fumigatus. Med Mycol 56: S83-S92, 2018.

30) Dunne K, Hagen F, Pomeroy N, Meis JF, Rogers TR:
Intercountry transfer of triazole-resistant Aspergillus fumigatus on plant bulbs. Clin Infect Dis 65: 147-149, 2017.

31) Verweij PE, Ananda-Rajah M, Andes D, et al: International expert opinion on the management of infection caused by azole-resistant Aspergillus fumigatus. Drug Resist Updat 2122: 30-40, 2015.

32) Hagiwara D, Takahashi H, Fujimoto M, Sugahara M, Misawa Y, Gonoi T, Itoyama S, Watanabe A, Kamei K: Multiple-azole resistant Aspergillus fumigatus harboring Cry51A TR46/ Y121F/T289A isolated in Japan. J Infect Chemother 22: 577$579,2016$.

33) Onishi K, Muhammad Sarumoh B, Hagiwara D, Watanabe A, Kamei K, Toyotome T: Azole-resistant Aspergillus fumigatus containing a 34-bp tandem repeat in cyp51A promoter is isolated from the environment in Japan. Med Mycol J 58: E67E70, 2017.

34) Tsuchido Y, Tanaka M, Nakano S, Yamamoto M, Matsumura Y, Nagao M: Prospective multicenter surveillance of clinically isolated Aspergillus species revealed azole-resistant Aspergillus fumigatus isolates with TR34/L98H mutation in the Kyoto and Shiga region of Japan. Med Mycol 57: 997-1003, 2019.

35) Nakano Y, Tashiro M, Urano R, Kikuchi M, Ito N, Moriya E, Shirahige T, Mishima M, Takazono T, Miyazaki T, Izumikawa $\mathrm{K}$ : Characteristics of azole-resistant Aspergillus fumigatus attached to agricultural products imported to Japan. J Infect Chemother 26: 1021-1025, 2020.

36) Tashiro M, Izumikawa K, Hirano K, et al: Correlation between triazole treatment history and susceptibility in clinically isolated Aspergillus fumigatus. Antmicrob Agents Chemother 56: 4870-4875, 2012.

37) Fisher MC, Hawkins NJ, Sanglard D, Gurr SJ: Worldwide emergence of resistance to antifungal drugs challenges human health and food security. Science 360: 739-742, 2018. 